\title{
Tenofovir vs. entecavir in reducing hepatocellular carcinoma risk in patients with chronic HBV infection? - Still an unsolved question
}

\author{
Hyun-Seok Kim ${ }^{1}$, Hashem B. El-Serag ${ }^{1,2}$ \\ ${ }^{1}$ Section of Gastroenterology and Hepatology, Baylor College of Medicine, Houston, TX, USA; ${ }^{2}$ U.S. Michael E. DeBakey Veterans Affairs Medical \\ Center, Houston, TX, USA \\ Correspondence to: Hashem B. El-Serag, MD, MPH. Section of Gastroenterology and Hepatology, Baylor College of Medicine, 7200 Cambridge \\ Street, Houston, Texas 77030, USA. Email: hasheme@bcm.edu. \\ Comment on: Yip TC, Wong VW, Chan HL, et al. Tenofovir Is Associated With Lower Risk of Hepatocellular Carcinoma Than Entecavir in Patients \\ With Chronic HBV Infection in China. Gastroenterology 2020;158:215-5.e6.
}

Submitted Apr 02, 2019. Accepted for publication Jun 08, 2020.

doi: 10.21037/hbsn-2020-3

View this article at: http://dx.doi.org/10.21037/hbsn-2020-3

Chronic hepatitis $\mathrm{B}$ virus (CHB) infection affects approximately 250 million persons worldwide (1). CHB is a major risk factor for liver failure, cirrhosis, and hepatocellular carcinoma (HCC), accounting for $45-55 \%$ of HCC cases (1). The incidence rates of HCC among CHB patients are $0.02-0.2$ per 100 person-years in inactive carriers, $0.3-0.6$ per 100 person-years in those without cirrhosis, and 2.2-3.7 per 100 person-years for those with compensated cirrhosis (2).

Currently, tenofovir [tenofovir disoproxil fumarate (TDF) and tenofovir alafenamide] and entecavir (ETV) are first-line monotherapies for CHB $(3,4)$. ETV, approved in 2005 by the FDA, is an oral nucleoside analogue with rare resistance in treatment-naïve patients $(3,4)$. TDF, which has been available since 2008 , is also a rapidly acting oral nucleotide analogue that has been shown to be highly effective in suppressing HBV replication $(3,4)$. The new version of tenofovir (Tenofovir alafenamide, TAF) can be used safely for those with renal or bone disease was FDA approved in $2016(3,4)$. However, HBV is rarely eradicated using either medication, and most patients with $\mathrm{HBV}$ infection require long term nucleos(t)ide analogue therapy.

There have been no head-to-head randomized clinical trials to compare the efficacy of ETV and tenofovir on longterm HCC risk in patients with CHB. Several observational studies examined these outcomes. In the January 2020 issue of Gastroenterology, Yip et al. published findings from a retrospective cohort study that reported TDF was associated with lower incidence of HCC compared to ETV in treatment-naive patients with chronic HBV in Hong Kong followed from 2008 to 2018 (5). There were 29,350 patients (mean age, $52.9 \pm 13.2$ years; $63.7 \%$ male); 1,309 were first treated with TDF (4.5\%) and 28,041 were first treated with ETV (95.5\%). Patients treated with TDF were younger (mean age, 43.2 vs. 53.4 years), and a lower proportion had cirrhosis $(2.9 \%$ vs. $13.6 \%)$ than patients treated with ETV. During a median follow-up time of 3.6 years (inter-quartile range, 1.7-5.0 years) after treatment began (mean 2.8 years in TDF and 3.7 years in ETV), 8 patients treated with TDF $(0.6 \%)$ and 1,386 patients treated with ETV (4.9\%) developed HCC as verified by diagnosis codes (155.0 HCC and 155.2 carcinoma of liver) or procedure codes for HCC treatment according to ICD9-CM codes. They used multiple statistical methods such as propensity score matching and weighting, inverse probability of treatment weighting (IPTW) and competitive risk analysis to minimize selection bias in the retrospective cohort study. The propensity score incorporated using 17 baseline characteristics (age, sex, cirrhosis, ascites, hepatic encephalopathy, HBV DNA, HBeAg status, ALT, total bilirubin, INR, platelet, albumin, creatinine, diabetes mellitus, hypertension, dialysis, and calendar year of treatment initiation). Patients treated with TDF had a lower risk of HCC than that in patients treated with ETV after propensity score weighting (hazard ratio, 0.36, 95\% CI, 0.16-0.80; $\mathrm{P}=0.013$ ) and $1: 5$ matching (hazard ratio, $0.39,95 \% \mathrm{CI}, 0.18-0.84 ; \mathrm{P}=0.016)$. The authors suggested a potential biological mechanism that a nucleotide such as 
TDF, compared to a nucleoside such as ETV, may elevate the interferon (IFN) lamda-3 level that further induces IFN-stimulated genes that can have anti-tumor effects and inhibit HBsAg production $(6,7)$.

Choi et al. was the first to report that TDF treatment group may have lower HCC incidence rates compared to the ETV treatment group (8). Their study included two retrospective cohorts of Korean patients with $\mathrm{HBV}$ infection; one from a large administrative dataset from January, 2010 to December, 2016 (24,156 HBV patients and 984 HCC incident cases), and the second from the tertiary hospital-based cohort from January 2010 to October 2017 (2,701 HBV patients and $154 \mathrm{HCC}$ incident cases). The results in both cohorts showed that those treated with TDF had about 39\% lower HCC incidence rate (hazard ratio $0.61,95 \% \mathrm{CI}, 0.54-0.70)$ than those treated with ETV. However, two additional studies from Korea that were published later did not show a statistically significant difference in HCC incidence rate between ETV and TDF groups $(9,10)$. Their study also used propensity score matching and IPTW analysis to minimize the effects of selection bias and confounding. Three abstracts presented at the 2019 AASLD annual meeting on this topic. Two European cohorts $(11,12)$ (PAGE-B cohort including about 2,000 Caucasian patients and a French cohort including 2,658 patients) also did not reveal significant difference in HCC risk between the two drugs, while a US retrospective cohort study (13) using a commercial insurance claims database supported lower HCC incidence in TDF group compared to ETV group (adjusted hazard ratio of 0.56 , $95 \%$ CI, 0.37-0.86). There are two meta-analyses in this topic, both published in 2019, with conflicting conclusions $(14,15)$. Wang et al. found no statistical difference between the two drugs in terms of HCC incidence, virological response, biochemical response, serological conversion, or drug resistance, while Zhang et al. study suggested that TDF treated group had a lower risk of HCC than ETV treated group (pooled hazard ratio $0.66,95 \%$ CI, $0.49-0.89$ ). Only three studies were common to both metaanalyses.

There are several points to make in interpreting these cohort findings properly. First, all studies were retrospective cohort design; thus, underlying biases and residual confounders could not be fully eliminated despite the use of propensity score and competitive risk analysis. The best way to correct biases is in the study design, not the analysis. In the Yip's paper, there were significant differences in baseline characteristics; those treated with TDF were younger with higher proportions of $\mathrm{HBeAg}$ positive, women, cirrhosis (2.9\% in TDF and $13.6 \%$ in ETV), hypertension or diabetes, and a lower HBV DNA, ALT, creatinine levels and therefore had lower baseline HCC risk based on the GAG-HCC score, CU-HCC score, PAGE-B score and REACH-B scores. Propensity score is the conditional probability of receiving treatment given all confounders (16). Among patients with the same propensity score, the treated and untreated group tend to have the equal distribution of measured confounders. However, propensity score is calculated based on only known, identified and available variables and these variables need to have level of accuracy and completeness (17). The propensity score in Yip's paper did not account for smoking status, alcohol use, HBV genotype, and adherence to HCC surveillance program or medication. Second, adjusting for the immortal time bias is essential in pharmacoepidemiological studies (18). Immortal time is defined as a period of follow-up during which, by design, death, or the study outcome cannot occur (18). In this context, immortal time is the time between the time of HBV acquisition and the treatment initiation. Since ETV was FDA approved earlier than TDF, the number of patients treated with TDF was significantly lower than the ETV group (1,309 TDF vs. 28,041 ETV) and had a shorter follow-up time to develop HCC (mean 2.8 years in TDF vs. 3.7 years in ETV). In order to control for the immortal time bias, knowing the date of HBV infection acquisition is crucial, which would be difficult to accurately obtain and while likely in infancy or childhood in most patients in the study. Third, over adjustment may also obscure significant findings (19). In order to adjust for the time-varying exposure to medications, Kim et al.'s study limited their cohort from 2012 when both drugs became reimbursed by the National Health Insurance Service in South Korea (9). Their results showed there was no significant difference between the two drugs in the incidence of HCC, liver transplant or death. Additionally, the follow-up duration in Yip's study (mean 2.8 years in TDF vs. 3.7 years in ETV) may not be sufficient to capture HCC incident cases, especially for those treated with TDF whose baseline HCC risk is low with only $2.9 \%$ being cirrhotic. Fourth, it is interesting that viral suppression by the two drugs at 1-year post-treatment was not a significant factor in HCC incidence in both Yip and Choi's papers. However, both studies showed a higher HBV DNA normalization rate in the TDF group, and Choi's study also showed a greater ALT normalization at 1-year post-treatment in the TDF group compared to the ETV group. Yip et al. published a 
different article in 2019 using the same Hong Kong cohort from 2005 to 2016 that showed complete viral suppression during the follow-up period to be associated with significantly lower HCC risk than those with incomplete viral suppression $(5.6 \%$ in complete suppression $v s .7 .8 \%$ in incomplete viral suppression at 8 years, adjusted hazard ratio 1.69; 95\% CI, 1.36-2.09) (20). The lack of statistical significance between viral suppression at 1 -year posttreatment and HCC incidence risk in the current Yip study could be due to missing values filled by multiple imputation. Moreover, indication for $\mathrm{HBV}$ treatment and the treating physicians' decision criteria to choose between the two drugs were unclear in the study. It is possible that the various indications for treatment or physician preference for the medication are related to baseline HCC risk. Lastly, most studies were conducted in East Asia, so generalizability to other countries needs to be elucidated.

In conclusion, the paper by Yip et al. adds another piece of evidence to an evolving, but yet inconclusive story that HBV patients treated with TDF may have a lower HCC risk compared to those treated with ETV. Further welldesigned prospective studies using a large sample size from different geographic regions and sufficient follow-up time are warranted to answer this important question.

\section{Acknowledgments}

Funding: None.

\section{Footnote}

Provenance and Peer Review: This article was commissioned by the editorial office, Hepatobiliary Surgery and Nutrition. The article did not undergo external peer review.

Conflicts of Interest: Both authors have completed the ICMJE uniform disclosure form (available at https://hbsn. amegroups.com/article/view/10.21037/hbsn-2020-3/coif). The authors have no conflicts of interest to declare.

Ethical Statement: The authors are accountable for all aspects of the work in ensuring that questions related to the accuracy or integrity of any part of the work are appropriately investigated and resolved.

Open Access Statement: This is an Open Access article distributed in accordance with the Creative Commons Attribution-NonCommercial-NoDerivs 4.0 International
License (CC BY-NC-ND 4.0), which permits the noncommercial replication and distribution of the article with the strict proviso that no changes or edits are made and the original work is properly cited (including links to both the formal publication through the relevant DOI and the license). See: https://creativecommons.org/licenses/by-nc-nd/4.0/.

\section{References}

1. GBD 2016 Disease and Injury Incidence and Prevalence Collaborators. Global, regional, and national incidence, prevalence, and years lived with disability for 328 diseases and injuries for 195 countries, 1990-2016: a systematic analysis for the Global Burden of Disease Study 2016. Lancet 2017;390:1211-59.

2. El-Serag HB. Epidemiology of viral hepatitis and hepatocellular carcinoma. Gastroenterology 2012;142:1264-73.e1.

3. Terrault NA, Lok ASF, McMahon BJ, et al. Update on prevention, diagnosis, and treatment of chronic hepatitis B: AASLD 2018 hepatitis B guidance. Hepatology 2018;67:1560-99.

4. European Association for the Study of the Liver. Electronic address eee, European Association for the Study of the L. EASL 2017 Clinical Practice Guidelines on the management of hepatitis B virus infection. J Hepatol 2017;67:370-98.

5. Yip TC, Wong VW, Chan HL, et al. Tenofovir Is Associated With Lower Risk of Hepatocellular Carcinoma Than Entecavir in Patients With Chronic HBV Infection in China. Gastroenterology 2020;158:215-25.e6.

6. Koike K, Suyama K, Ito H, et al. Randomized prospective study showing the non-inferiority of tenofovir to entecavir in treatment-naive chronic hepatitis B patients. Hepatol Res 2018;48:59-68.

7. Murata K, Asano M, Matsumoto A, et al. Induction of IFN-lambda3 as an additional effect of nucleotide, not nucleoside, analogues: a new potential target for HBV infection. Gut 2018;67:362-71.

8. Choi J, Kim HJ, Lee J, et al. Risk of Hepatocellular Carcinoma in Patients Treated With Entecavir vs. Tenofovir for Chronic Hepatitis B: A Korean Nationwide Cohort Study. JAMA Oncol 2019;5:30-6.

9. Kim SU, Seo YS, Lee HA, et al. A multicenter study of entecavir vs. tenofovir on prognosis of treatmentnaive chronic hepatitis B in South Korea. J Hepatol 2019;71:456-64.

10. Lee SW, Kwon JH, Lee HL, et al. Comparison of 
tenofovir and entecavir on the risk of hepatocellular carcinoma and mortality in treatment-naive patients with chronic hepatitis B in Korea: a large-scale, propensity score analysis. Gut 2020;69:1301-8.

11. Papatheodoridis GV. Similar risk of hepatocellular carcinoma (HCC) development during long-term entecavir (ETV) or tenofovir disoproxil fumarate (TDF) therapy in Caucasian chronic hepatitis $\mathrm{B}(\mathrm{CHB})$ patients. Hepatology 2019;70:286A.

12. Group PSAAHS. Tenofovir versus entecavir in HBV chronic infection: impact on HCC and other liver-related complications occurrences. Hepatology 2019;70:129A.

13. Kim WR. Risk of incident hepatocellular carcinoma in hepatitis B-infected patients treated with tenofovir disoproxil fumarate versus entecavir: a US administrative claims analysis. Hepatology 2019;70:302A-3A.

14. Wang X, Liu X, Dang Z, et al. Nucleos(t)ide Analogues for Reducing Hepatocellular Carcinoma in Chronic Hepatitis B Patients: A Systematic Review and Meta-Analysis. Gut Liver 2020;14:232-47.

15. Zhang Z, Zhou Y, Yang J, et al. The effectiveness of TDF versus ETV on incidence of $\mathrm{HCC}$ in $\mathrm{CHB}$ patients: a meta analysis. BMC Cancer 2019;19:511.

16. Little RJ, Rubin DB. Causal effects in clinical and epidemiological studies via potential outcomes: concepts and analytical approaches. Annu Rev Public Health 2000;21:121-45.

17. Austin PC. An Introduction to Propensity Score Methods for Reducing the Effects of Confounding in Observational Studies. Multivariate Behav Res 2011;46:399-424.

18. Levesque LE, Hanley JA, Kezouh A, et al. Problem of immortal time bias in cohort studies: example using statins for preventing progression of diabetes. BMJ 2010;340:b5087.

19. Schisterman EF, Cole SR, Platt RW. Overadjustment bias and unnecessary adjustment in epidemiologic studies. Epidemiology 2009;20:488-95.

20. Yip TC, Wong GL, Chan HL, et al. HBsAg seroclearance further reduces hepatocellular carcinoma risk after complete viral suppression with nucleos(t)ide analogues. J Hepatol 2019;70:361-70.
Cite this article as: Kim HS, El-Serag HB. Tenofovir vs. entecavir in reducing hepatocellular carcinoma risk in patients with chronic HBV infection?-Still an unsolved question. HepatoBiliary Surg Nutr 2021;10(1):119-122. doi: 10.21037/ hbsn-2020-3 